a crucial role in the development of obesityrelated insulin resistance. J Clin Invest. 2003 ; 112(12):1821-1830.

5. Kosteli A, et al. Weight loss and lipolysis promote a dynamic immune response in murine adipose tissue. J Clin Invest. 2010;120(10):3466-3479.

6. Shoelson SE, Lee J, Goldfine AB. Inflammation and insulin resistance. J Clin Invest. 2006; 116(7):1793-1801.

7. Hotamisligil GS, Shargill NS, Spiegelman BM. Adipose expression of tumor necrosis factor-alpha: direct role in obesity-linked insulin resistance. Science. 1993;259(5091):87-91.

8. Lumeng CN, Bodzin JL, Saltiel AR. Obesity induces a phenotypic switch in adipose tissue macrophage polarization. J Clin Invest. 2007;117(1):175-184

9. Kang K, et al. Adipocyte-derived Th2 cytokines and myeloid PPARdelta regulate macrophage polarization and insulin sensitivity. Cell Metab. 2008;7(6):485-495.
10. Odegaard JI, et al. Macrophage-specific PPARgamma controls alternative activation and improves insulin resistance. Nature. 2007;447(7148):1116-1120.

11. Odegaard JI, et al. Alternative M2 activation of Kupffer cells by PPARdelta ameliorates obesity-induced insulin resistance. Cell Metab. 2008; 7(6):496-507.

12. Olefsky J, Glass C. Macrophages, inflammation, and insulin resistance. Annu Rev Physiol. 2010; 72:219-246.

13. Nishimura S, et al. CD8+ effector T cells contribute to macrophage recruitment and adipose tissue inflammation in obesity. Nat Med. 2009; 15(8):914-920

14. Winer $\mathrm{S}$, et al. Normalization of obesity-associated insulin resistance through immunotherapy. Nat Med. 2009;15(8):921-929.

15. Liu J, et al. Genetic deficiency and pharmacological stabilization of mast cells reduce diet-induced obesity and diabetes in mice. Nat Med. 2009;
15(8):940-945

16. Cancello R, et al. Reduction of macrophage infiltration and chemoattractant gene expression changes in white adipose tissue of morbidly obese subjects after surgery-induced weight loss. Diabetes. 2005; 54(8):2277-2286.

17. Yvan-Charvet L, Wang N, Tall AR. Role of HDL, $\mathrm{ABCA} 1$, and $\mathrm{ABCG} 1$ transporters in cholesterol efflux and immune responses. Arterioscler Thromb Vasc Biol. 2010;30(2):139-143.

18. Bechah Y, Paddock CD, Capo C, Mege JL, Raoult D. Adipose tissue serves as a reservoir for recrudescent Rickettsia prowazekii infection in a mouse model. PLoS One. 2010;5(1):e8547.

19. Combs TP, et al. The adipocyte as an important target cell for Trypanosoma cruzi infection. J Biol Chem. 2005;280(25):24085-24094.

20. Neyrolles O, et al. Is adipose tissue a place for Mycobacterium tuberculosis persistence? PLoS One. 2006;1:e43.

\title{
c-Maf and you won't see fat
}

\author{
Laurie K. McCauley
}

Department of Periodontics and Oral Medicine, School of Dentistry, and Department of Pathology, Medical School, University of Michigan, Ann Arbor, Michigan, USA.

\begin{abstract}
Osteoporosis is a common, age-related bone disease that results from an imbalance between the processes of bone formation and bone resorption, resulting in reduced bone mass and increased risk of fracture. Mesenchymal stem cells have the capacity to differentiate into osteoblastic and adipogenic lineages; recent research suggests that the switch between these two fates may be key to the decreased bone density that occurs with aging. In this issue, Nishikawa et al. demonstrate that the basic leucine-zipper transcription factor Maf (also known as c-Maf) is central to osteoblast lineage commitment. In addition, they find that increased oxidative stress - as occurs with aging - decreases Maf expression. This work advances understanding of the transcriptional regulation of cell fate decisions and may help direct the development of new therapies to fight age-related bone loss.
\end{abstract}

Osteoporosis is a metabolic bone disease that results from an imbalance between the processes of bone formation and bone resorption, leading to reduced bone mass and increased susceptibility to fracture. Osteoporosis is commonly age associated, and one out of two women and one out of four men over the age of 50 will experience an osteoporotic fracture during their lifetime, with patient care costs estimated at $\$ 14$ billion per year $(1,2)$. Recent research has identified a correlative relationship of fat deposition in bone marrow with bone density. Many but not all studies link the two in an inverse relationship that suggests marrow fat may be driven at the

Conflict of interest: The author has declared that no conflict of interest exists.

Citation for this article: J Clin Invest. 2010; 120(10):3440-3442. doi:10.1172/JCI44786 expense of new bone formation. While the role of fat in bone remains unclear, current thinking supports a reciprocal relationship between adipogenesis and osteogenesis governed by mesenchymal stem cell (MSC) lineage allocation.

The bone marrow houses a wide variety of cell types at various stages of differentiation. Cells of the mesenchymal lineage reside in the marrow and give rise to osteoblasts and adipocytes, among other cell types (3). Over the past decade, a considerable effort has been devoted toward understanding how MSC fates can be directed toward adipogenic and osteogenic lineages. The critical determinants of this switch are unclear, but a complete understanding of the process may direct the development of therapies for age-associated bone loss. The mutual exclusivity of the osteoblast and adipocyte cell fates suggests that sig- nals that direct cells down one lineage may prevent them from traversing down the other. Prime examples of these switch signals are CCAAT/enhancer binding protein (C/EBP), a trigger for adipogenesis; PPAR $\gamma$, which promotes adipocyte maturation; and Runx2, an osteoblastic transcriptional mediator (4). Impaired PPAR $\gamma$ signaling shifts the fate of MSCs in the marrow toward the osteoblast lineage (5). The wnt pathway can suppress PPAR $\gamma$, favoring MSC differentiation to osteoblasts (6). Other transcriptional mediators associated with osteoblast/adipocyte specification include $\Delta \mathrm{FosB}, \mathrm{TAZ}$, Esr1, Msx2, C/EBP $\beta$, and Id4, though their specificity as determinants of the age-related switch in bone is unclear $(7,8)$.

\section{Identification of Maf as a cell fate switch factor}

In this issue, Nishikawa et al. demonstrate, in an elegant series of studies, that the basic leucine-zipper (bZIP) transcription factor Maf (musculoaponeurotic fibrosarcoma, also known as c-Maf) is central to osteoblast lineage commitment with age (9) (Figure 1). Via a genome-wide screening of transcription factors expressed in cells derived from calvaria, Maf was identified as a factor that increased more than 4-fold during osteoblastogenesis. It was further found to be highly expressed in bone marrow stromal cells (BMSCs) and reduced in 


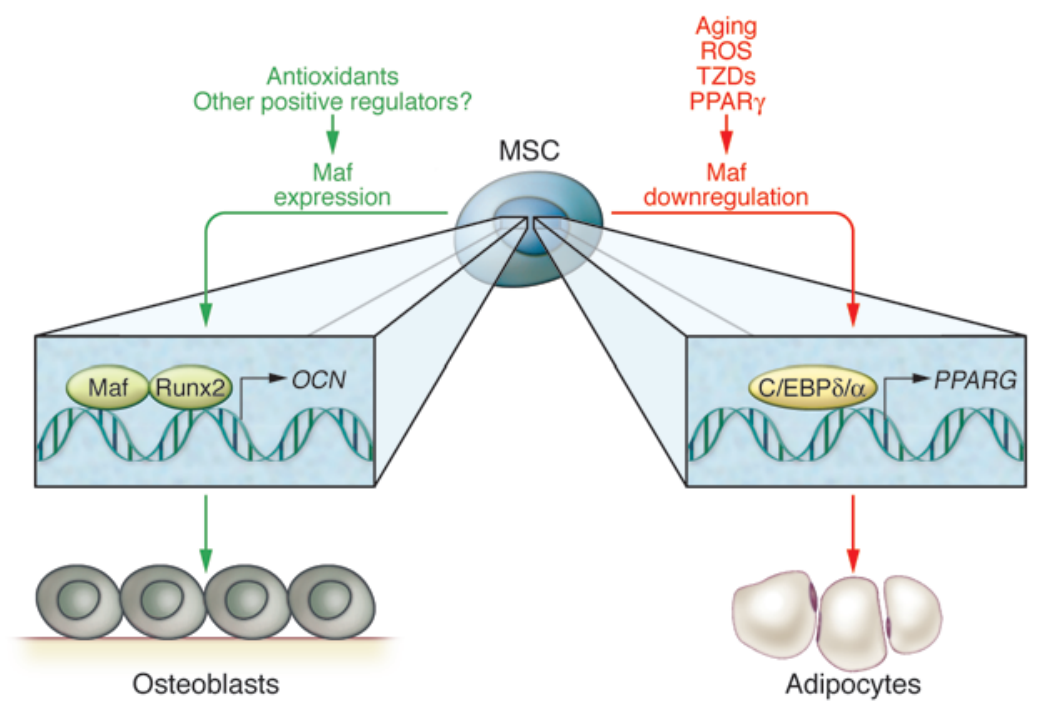

Figure 1

Maf expression determines whether MSCs differentiate toward the adipocyte or osteoblast lineage. The transcription factor Maf partners with Runx2 to regulate osteoblastic genes, such as osteocalcin $(O C N)$. Maf is downregulated during aging and under the influence of reactive oxygen species (ROS) and thiazolidinediones (TZDs), pharmaceutical drugs for the treatment of diabetes. Downregulation of Maf leads to increased PPARG through the activation of C/EBP $/ \alpha$ and leads to increased adipogenesis. PPARG itself also downregulates Maf, promulgating a cycle of adipogenesis. Antioxidants promote Maf expression and reverse the adipogenic phenotype, resulting in greater bone formation. Other factors that increase Maf may provide promising therapeutic strategies for the treatment of age-associated bone loss.

expression in BMSCs during aging (32- vs. 8-week-old mice).

The Maf transcription factor was originally identified as a retina-specific factor and a $\mathrm{T}$ helper cell factor responsible for IL-4 production $(10,11)$. The first description of its deletion in mice indicated runting that was attributed to a cartilage phenotype (11). Maf is a member of the group of large Maf family members characterized by the presence of the bZIP domain (12). The bZIP domain consists of a region rich in basic residues, followed by heptad repeats of hydrophobic residues, forming a leucine zipper. A conserved amino-terminal domain in the large Maf proteins is rich in Asp, Glu, Ser, Thr, and Pro residues, which are targets for phosphorylation that likely regulate Maf function (13). Several groups of bZIP proteins have been well described and have prominent roles in bone biology, including the AP- 1 and CREB/ATF families (14). These other bZIP proteins bind relatively small consensus sequences of 7-8 bp in length, whereas the Maf proteins bind longer consensus sequences of 13-14 bp, termed Maf-recognition elements (MAREs) (12). This study by Nishikawa et al. found 5 MARE sequences in the osteocalcin promoter and demonstrated a physical inter- action between Maf and Runx2 on the osteocalcin gene.

A series of experiments with $\mathrm{Maf}^{-1}$ and heterozygous mice unraveled the downstream mechanisms of Maf in the skeleton. Because $\mathrm{Maf}^{-1}$ mice suffer perinatal lethality, studies focused on early osteogenesis. Bone formation was severely impaired with low alkaline phosphatase and osteocalcin gene expression. Interestingly, Runx2 expression and rates of osteoblast proliferation and apoptosis were not altered. Since Maf deletion was previously found to result in a severe cartilage phenotype, the impact of the cartilaginous development on osteogenesis was questioned. Defective bone formation in intramembraneous bones along with cell-autonomous osteoblast differentiation defects in vitro suggest that the role of Maf in skeletal development could not be explained entirely by the cartilage phenotype. Instead, the in vitro cell studies pointed to Maf as a critical determinant of adipogenic (e.g., PPAR $\gamma, \mathrm{C} / \mathrm{EBP} \alpha$, and FABP4) versus osteoblastogenic (alkaline phosphatase, osteocalcin) gene expression. Furthermore, studies with $\mathrm{Maf}^{\mathrm{t}-\text { - }}$ mice revealed reduced bone formation and increased numbers of bone marrow adipocytes with age in the absence of a cartilage phenotype. This bone phenotype was rescued with the addition of osteoblastic cells overexpressing Maf.

\section{Regulation of the regulator}

Nishikawa et al. also attempted to identify the factors responsible for the age-related decrease in Maf levels. Several candidate genes previously shown to be associated with aging, such as $B m p 2, T g f b, I l 11$, and Igf1, were evaluated for their ability to mediate Maf expression, but none were found to significantly regulate it. Reactive oxygen species were found to decrease Maf, and, furthermore, in vivo administration of the antioxidant $\mathrm{N}$-acetylcysteine could rescue the loss of bone mass associated with Maf haploinsufficiency. Upstream modulation of Maf was found by PPAR $\gamma$ and p53, raising the possibility that therapeutics that regulate this pathway may have potential for the treatment of age-associated bone loss. Interestingly, a recent study suggests that cells of the osteogenic lineage may be more susceptible to oxidative stress triggered by PPAR $\gamma$, adding to the complexity of the cell fate (15).

\section{Future directions and therapeutic implications}

The studies by Nishikawa et al. raise important questions and suggest exciting new therapeutic potentials. What are the factors or cues that positively regulate Maf and could be used as therapeutic strategies? The potential of Maf to be a target of mechanical stimuli may be of interest, as such stimuli have been found to regulate the bone marrow mesenchymal switch from an adipogenic to an osteoblastic fate (16). Since Runx2 phosphorylation has been found to be critical for the regulation of osteoblast differentiation (17), will phosphorylation sites in the amino-terminal domain of Maf be critical for its ability to interact with Runx2 and/or impact osteoblast/adipocyte lineage determination? What are the implications of turning on Maf in the mesenchymal cell lineage? Data in the study by Nishikawa suggested that loss of Maf did not alter calvarial cell proliferation or apoptosis. If it bears out that positive regulation of Maf does not lead to a proliferative osteoblast phenotype, it may offer an attractive alternative therapeutic approach to other targeted anabolics whose alleged association with increased risk of osteosarcoma could limit enthusiasm for their use.

The impact of aging and the decline in Maf found in the study by Nishikawa et al. 
represent what I believe to be a novel consideration of osteoblast regulation. If Runx2 levels remain fairly consistent with age, yet osteoblastic differentiation is reduced, the concept of a controller or modifier for the master regulator is intriguing. Considering the murine lifespan, the age dependence of Maf expression and activity also requires further investigation using standard models of aging and going beyond the age of 32 weeks used by Nishikawa et al. It would also be of interest to see the role of Maf in the same animal, with differing sites of fatty versus hematopoietic marrow. Finally, and most importantly, these exciting studies require thorough evaluation and validation in humans, including studies with drugs that impact bone and marrow fat via careful analysis of their impact on Maf. Thiazolidinediones are antidiabetic drugs associated with an increase in marrow adiposity and reduced bone $(18,19)$. Nishikawa and colleagues suggest that these drugs act at least in part by reducing Maf expression, which inhibits PPAR $\gamma$ in the MSC lineage to direct cells toward adipogenesis. As MSC therapies become more common, optimizations through altering transcriptional regulation of cell differentiation will become more important and more viable (20). This study provides what I believe to be an exciting new discovery that may lead to new strategies for the treatment of the devastating consequences of age-associated bone loss.

\section{Acknowledgments}

The author thanks Renny Franceschi and Laura McCabe for input. Related research in the author's laboratory is supported by NIH/NIDDK grant RO1 DK53904.

Address correspondence to: Laurie K. McCauley, University of Michigan, $1011 \mathrm{~N}$. University Ave., Room 3397, Dept. POM, Ann Arbor, Michigan 48109, USA. Phone: 734.647.3206; Fax: 734.763.5503; E-mail: mccauley@umich.edu.

1. NIH Osteoporosis and Related Bone Diseases National Resource Center. Osteoporosis Overview Web Site. http://www.niams.nih.gov/Health_Info/ Bone/Osteoporosis/. Accessed August 17, 2010.

2. Compston JE. Osteoporosis: social and economic impact. Radiol Clin North Am. 2010;48(3):477-482.

3. Bianco P, Robey PG, Simmons PJ. Mesenchymal stem cells: revisiting history, concepts, and assays. Cell Stem Cell. 2010;2(4):313-319.

4. Kawai M, Sousa KM, MacDougald OA, Rosen CJ. The many facets of PPAR $\gamma$ : novel insights for the skeleton. Am J Physiol Endocrinol Metab. 2010;299(1):E3-E9.

5. Akune T, et al. PPAR $\gamma$ insufficiency enhances osteogenesis through osteoblast formation from bone marrow progenitors. J Clin Invest. 2004;113(6):846-855.

6. Kang S, Bennett CN, Gerin I, Rapp LA, Hankenson $\mathrm{KD}$, MacDougald OA. Wnt signaling stimulates osteoblastogenesis of mesenchymal precursors by suppressing CCAAT/enhancer-binding protein alpha and peroxisome proliferator-activated receptor gamma. J Biol Chem. 2007;282(19):14515-14524.

7. Takada I, Kouzmenko AP, Kato S. Wnt and PPAR $\gamma$ signaling in osteoblastogenesis and adipogenesis. Nat Rev Rheumatol. 2009;5(8):442-447.

8. Tokuzawa Y, et al. Id4, a new candidate gene for senile osteoporosis, acts as a molecular switch promoting osteoblast differentiation. PLOS Genetics. 2010; 6(7):e1001019.
9. Nishikawa K, et al. Maf promotes osteoblast differentiation in mice by mediating the age-related switch in mesenchymal cell differentiation. J Clin Invest. 2010;120(10):3455-3465.

10. Ho IC, Hodge MR, Rooney JW, Glimcher LH. The proto-oncogene c-maf is responsible for tissue-specific expression of interleukin-4. Cell. 1996;85(7):973-983.

11. Kim JI, Li T, Ho IC, Grusby MJ, Glimcher LH. Requirement for the c-Maf transcription factor in crystallin gene regulation and lens development. Proc Natl Acad US A. 1999;96(7):3781-3785.

12. Blank V, Andrews NC. The Maf transcription factors: regulators of differentiation. Trends Biochem Sci. 1997;22(11):437-441.

13. Kataoka K. Multiple mechanisms and functions of Maf transcription factors in the regulation of tissue-specific genes. J Biochem. 2007;141(6):775-781.

14. Wagner EF, Eferl R. Fos/AP-1 proteins in bone and the immune system. Immunol Rev. 2005;208:126-140.

15 . Bruedigam $C$, et al. A new concept underlying stem cell lineage skewing that explains the detrimental effects of thiazolidinediones on bone. Stem Cells. 2010;28(5):916-927.

16. Sen B, Xie Z, Case N, Ma M, Rubin C, Rubin J. Mechanical strain inhibits adipogenesis in mesenchymal stem cells by stimulating a durable beta-catenin signal. Endocrinology. 2008;149(12):6065-6075.

17. Ge C, et al. Identification and functional characterization of ERK/MAPK phosphorylation sites in the Runx2 transcription factor. J Biol Chem. 2009; 284(47):32533-32543.

18. Gimble JM, et al. Peroxisome proliferator-activated receptor-gamma activation by thiazolidinediones induces adipogenesis in bone marrow stromal cells. Mol Pharmacol. 1996;50(5):1087-1094.

19. Nuttall ME, Patton AJ, Olivera DL, Nadeau DP, Gowen M. Human trabecular bone cells are able to express both osteoblastic and adipocytic phenotype: implications for osteopenic disorders. J Bone Miner Res. 1998;13(3):371-382.

20. Undale AH, Westendorf JJ, Yaszemski MJ, Khosla S. Mesenchymal stem cells for bone repair and metabolic bone diseases. Mayo Clin Proc. 2009;84(10):893-902. 